\title{
Conformational disorder in vitreous systems probed by photoluminescence activity in $\mathrm{SiO}_{2}$
}

\author{
M. Leone, S. Agnello, R. Boscaino, M. Cannas, and F. M. Gelardi \\ Istituto Nazionale per la Fisica della Materia and Department of Physical and Astronomical Sciences, University of Palermo, \\ Via Archirafi, 36, I-90123 Palermo, Italy
}

(Received 22 March 1999)

\begin{abstract}
The emission bands $\alpha_{E}(4.2 \mathrm{eV})$ and $\beta(3.1 \mathrm{eV})$ have been investigated in a variety of as-grown natural silica types. We report experimental results on the stationary photoluminescence spectra and their relationship with the absorption band $B_{2 \beta}(5.15 \mathrm{eV})$, on the temperature dependence of their competition, on their excitation pathway and their kinetic decay ensuing a pulse excitation. We use our results to characterize the electronic transitions involved and the intersystem crossing process linking the two excited states. Our results evidence the role played by the conformational disorder. In particular, we make use of a model in which the interconversion processes between conformational substates are effective at high temperature $(T>200 \mathrm{~K})$ and become frozen at low temperature $(T<120 \mathrm{~K})$. Finally we discuss our results on the basis of a structural model ascribing these optical activities to an oxygen deficient center related to a Ge impurity. [S0163-1829(99)03540-7]
\end{abstract}

\section{INTRODUCTION}

The spectral properties of natural silica $\left(a-\mathrm{SiO}_{2}\right)$, as optical absorption (OA) bands and photoluminescence (PL) emissions, are affected by several kinds of point defects. Even if these defects have been the subject of widespread investigation for more than three decades, many aspects of their structure are not thoroughly understood and the relations between optical bands and specific defects are still matter of controversy (see Ref. 1 for a recent review). In particular, the interplay between the electronic properties of the point defect and the structural and dynamical properties of its surrounding appears to be of relevant interest. In this respect, the analysis of the spectral features in different silica types can yield insight on the nature of the chromophores responsible for the observed optical activity. Moreover, the thermal behavior, in a wide temperature range, of the overall spectral features can be studied for evidencing the role played by the vitreous matrix.

In previous papers, ${ }^{2-5}$ we studied the optical activity of two types of oxygen-deficient centers (ODC's) of the $\mathrm{SiO}_{2}$ network, the former present in the pristine material and the latter induced by $\gamma$ irradiation. The intrinsic point defects have an $\mathrm{OA}$ band at $5.02 \mathrm{eV}\left(B_{2 \alpha}\right)$ and an emission band, labeled as $\alpha_{I}$, at about $4.4 \mathrm{eV}$ (type- $A$ activity). On the other hand, the $\gamma$ rays induced centers present a PL band, labeled as $\alpha_{R}$, slightly but significantly different from the $\alpha_{I}$ for the line shape and for the shorter decay time. These data, together with a detailed analysis of the thermal behavior of the spectral moments, ${ }^{6}$ suggest that the two chromophores are structurally similar but for a different environment, probably due to the different conditions in which they are generated.

In this paper, we extend our previous study to the socalled type- $B$ activity, present in several types of natural silica and consisting in an OA band centered at $5.15 \mathrm{eV}\left(B_{2 \beta}\right.$ band) and two PL emissions centered at $4.2 \mathrm{eV}\left(\alpha_{E}\right.$ emission) and at $3.1 \mathrm{eV}(\beta$ emission $) .^{7-14}$ The interest in this activity is twofold. On the one hand, the structure of the active center is not yet established unambiguously, even if it is usually associated to an ODC involving a Ge impurity, either substitutional or localized in its proximity. ${ }^{7-12}$ On the other hand, some peculiarities of the PL emissions $\alpha_{E}$ and $\beta$ make them particularly suitable for studying the effects of the matrix disorder on the electronic properties of the active center. In fact, the two PL bands are assigned to the singletsinglet $\left(S_{1} \rightarrow S_{0}\right)$ and to the triplet-singlet $\left(T_{1} \rightarrow S_{0}\right)$ emission of the same center and the two excited states are linked by an intersystem crossing (ISC) process, which is expected to depend strongly on the temperature. In this scenario, the experimental investigation of this temperature dependence can yield information on the dynamical properties and the conformational disorder of the environment of the active center.

In the present paper, we study firstly the correlation between the two PL bands and the $B_{2 \beta}$ OA band in various types of natural silica, commercially available, with a nominal Ge content of the order of $1 \mathrm{ppm}$, to definitively verify that a single-point defect is responsible for the whole $B$-type activity. Secondly, we analyze in detail the dependence of the PL emissions on the temperature and on the fine tuning of the excitation energy within the $B_{2 \beta}$ OA band. This investigation aims to put in evidence how the structural and dynamical properties of the matrix and the conformational heterogeneity of the environment affect the spectral features of the optical active centers.

\section{METHODS AND MATERIALS}

\section{A. Outline of the experiments}

To outline our experiments and to make clear their discussion, we consider a simplified picture of the interaction of the exciting light with an ensemble of noninteracting centers having the following energy level diagram: the fundamental state $S_{0}$ and two excited states, a singlet $S_{1}$ and a triplet $T_{1}$, with energy $E_{S}$ and $E_{T}$, respectively. The monochromatic light (with energy $E_{\text {Exc }}$ ) is turned to excite the $S_{0} \rightarrow S_{1}$ transition and we assume that the absorption is low enough to guarantee the uniform intensity within the sample (low- 
extinction limit). Moreover, we disregard all the nonradiative relaxation from the excited states except the ISC process linking the states $S_{1}$ and $T_{1}$.

Within these approximations, the populations $n_{S}$ and $n_{T}$ of the states $S_{1}$ and $T_{1}$, respectively, obey the rate equations:

$$
\begin{gathered}
\frac{d}{d t} n_{S}=I\left(E_{\mathrm{Exc}}\right)-\left(k_{S}+k_{\mathrm{ISC}}\right) n_{S} \\
\frac{d}{d t} n_{T}=k_{\mathrm{ISC}} n_{S}-k_{T} n_{T},
\end{gathered}
$$

where $I\left(E_{\mathrm{Exc}}\right)$ is the rate of light absorption and $k_{\mathrm{SC}}$ is the rate of the ISC process. The decay constants $k_{S}$ and $k_{T}$ refer to the radiative decay from $S_{1}$ and from $T_{1}$ to $S_{0}$, namely to the $\alpha_{E}$ and to the $\beta$ emissions, respectively.

Under c.w. excitation, $n_{S}$ and $n_{T}$ reach their stationary values

$$
\begin{gathered}
n_{S}=\frac{I\left(E_{\mathrm{Exc}}\right)}{k_{S}+k_{\mathrm{ISC}}} \\
n_{T}=\frac{I\left(E_{\mathrm{Exc}}\right) k_{\mathrm{ISC}}}{k_{S}+k_{\mathrm{ISC}} k_{T}} .
\end{gathered}
$$

In these conditions the intensities of the stationary PL emissions from $S_{1}$ and $T_{1}$ are

$$
\begin{aligned}
& S\left(E_{\mathrm{Exc}}, E_{\mathrm{Em}}\right)=n_{S} k_{S} g_{S}\left(E_{\mathrm{Em}}\right)=I\left(E_{\mathrm{Exc}}\right) \frac{k_{S}}{k_{S}+k_{\mathrm{ISC}}} g_{S}\left(E_{\mathrm{Em}}\right) \\
& T\left(E_{\mathrm{Exc}}, E_{\mathrm{Em}}\right)=n_{T} k_{T} g_{T}\left(E_{\mathrm{Em}}\right)=I\left(E_{\mathrm{Exc}}\right) \frac{k_{\mathrm{ISC}}}{k_{S}+k_{\mathrm{ISC}}} g_{T}\left(E_{\mathrm{Em}}\right),
\end{aligned}
$$

where the normalized distributions $g_{S}\left(E_{\mathrm{Em}}\right)$ and $g_{T}\left(E_{\mathrm{Em}}\right)$ describe the energy spreading of the levels $E_{S}$ and $E_{T}$, peaked at $E_{S}=4.2 \mathrm{eV}$ ( $\alpha_{E}$ band) and at $E_{T}=3.1 \mathrm{eV}$ ( $\beta$ band), respectively.

We note that, in this oversimplified picture, the PLemission spectra are expected to depend on the exciting light energy only for their intensities, neither for their line shape nor for their spectral position. A similar expectation holds as well for the photoluminescence excitation (PLE) spectra, when we consider their variation with the detected light energy $E_{\mathrm{Em}}$. Moreover, we note that, according to Eq. (3), identical excitation spectra are expected for both emissions.

The comparison between the two PL emissions is conveniently carried out in terms of the ratio $\eta$ of their integrated intensities, that is, according to Eq. (3), $\eta=k_{S} / k_{\text {ISC }}$. In particular we will investigate the temperature dependence of $\eta$, which images the temperature dependence of the rate $k_{\text {ISC }}$.

Finally, if the exciting light is abruptly turned off (pulsed excitation), according to Eq. (1), the PL emission $\alpha_{E}$ will decay following the single exponential law:

$$
S(t)=S(0) \exp \left[-\left(k_{S}+k_{I S C}\right) t\right] .
$$

The decay of the emission $\beta$ is, in principle, somewhat more complicated, due to the time-dependent term $k_{\mathrm{ISC}} \cdot n_{S}$ in Eq. (1). However, due to the condition $k_{T} \ll k_{\text {ISC }}, k_{S}$ (well verified in our experiments), the solution simplifies to

$$
T(t)=T(0) \exp \left[-k_{T} t\right] .
$$

In the following sections, Eqs. (3)-(5) will be used as a framework for discussing the experimental results. Several discrepancies will be found and will be ascribed to those effects that are not taken into account by the simplified model, which these equations rely on, and in particular to the dynamics of the environment and to the inhomogeneous distribution of the $k_{\text {ISC }}$ rate.

\section{B. Experimental methods and samples}

PL spectra resulting from excitation in the wavelength range $220-270 \mathrm{~nm}(5.6-4.6 \mathrm{eV})$ were obtained using a spectrofluorometer Jasco PF-770, mounting a Xenon lamp of $150 \mathrm{~W}$. The samples were mounted in the holder in the socalled $45^{\circ}$ back-scattering geometry. The energy dependence of the detection system was preliminarily determined by placing a $\mathrm{MgO}$ scatterer in the sample position and performing a synchronous spectrum of both monochromators. Moreover, the excitation spectra were corrected for the spectral efficiency of the exciting light by using a Rhodamine $B$ sample in glycerol as a reference. ${ }^{15}$

For low-temperature measurements, we used an Oxford Optistat $^{\mathrm{CF}}$ continuous-flow helium cryostat, equipped with four optical windows; the temperature was controlled by an Oxford ITC503 instrument. At the required temperature, after $10 \mathrm{~min}$ for thermal equilibrium, spectra were recorded at $0.5 \mathrm{~nm}$ wavelength intervals.

Unless otherwise specified, the PL emissions were excited by light at $\lambda_{\mathrm{Exc}}=248 \mathrm{~nm}(5.0 \mathrm{eV})$, very close to the maximum of $B_{2 \beta}$ OA band, with an excitation bandwidth $\Delta \lambda_{\mathrm{Exc}}=5.0 \mathrm{~nm}$ and detected with a bandwidth $\Delta \lambda_{E m}$ $=1.5 \mathrm{~nm}$. The related PLE spectra were also measured, by detecting the emission intensity at the wavelength of the maximum of each PL band, with $\Delta \lambda_{\mathrm{Exc}}=1.5 \mathrm{~nm}$ and $\Delta \lambda_{\mathrm{Em}}=3.0 \mathrm{~nm}$. For both kinds of spectra the scan rate was $20 \mathrm{~nm} / \mathrm{min}$.

The fine tuning of the PL emissions was investigated by detecting the emission spectra at different $\lambda_{\text {Exc }}$, varied from 220 to $270 \mathrm{~nm}$ by steps of $1.5 \mathrm{~nm}$. For these measurements $\Delta \lambda_{\text {Exc }}$ was fixed to $3.0 \mathrm{~nm}$. The corresponding PLE spectra were measured at different $\lambda_{\mathrm{Em}}$ within the $\alpha_{E}$ and $\beta$ bands, scanning from 270 to $330 \mathrm{~nm}$ and from 360 to $435 \mathrm{~nm}$, respectively, by steps of $1.5 \mathrm{~nm}$.

Absorption spectra in the UV region were obtained on a spectrophotometer Cary Mod.2300, using a bandwidth of 0.5 $\mathrm{nm}$, a scan speed $0.5 \mathrm{~nm} / \mathrm{s}$ and a time constant of $1 \mathrm{~s}$. For low-temperature absorption spectra, a homemade optical Dewar was used, in which the temperature can be lowered by flushing with liquid nitrogen or helium.

The time decay of the transient PL $\alpha_{E}$ was measured using the synchrotron radiation (SR) at the superlumi experimental station on the I-beamline of HASYLAB at DESY (Hamburg, Germany), under single-bunch operation, by scanning $192 \mathrm{~ns}$ between adjacent SR pulses, $0.5 \mathrm{~ns}$ wide, under excitation at $5.0 \mathrm{eV}$. Measurements were carried out at 300 and $10 \mathrm{~K}$, using a helium-flow cryostat.

The measurements reported here were carried out in several types of natural silica, all of commercial origin. The list includes: Puropsil (QPA), EQ 906, EQ 912, ${ }^{16}$ Infrasil 301 

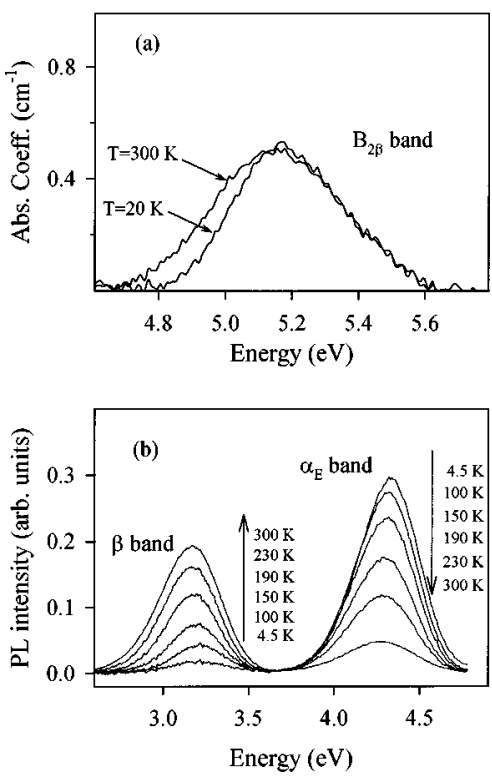

FIG. 1. (a) The isolated $B_{2 \beta}$ contribution to the optical absorption spectra of our sample I301 at 300 and $20 \mathrm{~K}$; (b) PL spectra of the same sample in the energy range $2.5 \div 5.0 \mathrm{eV}$ with excitation energy of $5.0 \mathrm{eV}$ at various temperatures, from 4.5 to $300 \mathrm{~K}$.

(I301), Herasil 1 (H1), Herasil 3 (H3), Homosil (HM), ${ }^{17}$ Vitreosil (VTS). ${ }^{18}$ Puropsil, EQ silica, and Infrasil are dry or type-I silica ([OH] content less than $15 \mathrm{ppm})$; all other materials are wet or type-II ([OH] content of nearly $200 \mathrm{ppm})$. All the samples are sized at $5 \times 5 \times 1 \mathrm{~mm}^{3}$ with the major surfaces optically polished and were used for our measurements as supplied.

\section{RESULTS AND DISCUSSION}

\section{A. Characterization of spectral properties}

All the investigated samples present the OA band centered at $5.15 \mathrm{eV}$ ( $B_{2 \beta}$ band). A typical OA spectrum is reported in Fig. 1(a), as detected in our sample I301 at high and low temperature. The band profile remains essentially the same on lowering the temperature down to $20 \mathrm{~K}$, apart from a narrowing and a slight blueshift.

Two well-resolved PL bands, centered at 3.1 and $4.2 \mathrm{eV}$ and both featuring temperature-dependent intensities, are excited within the $B_{2 \beta}$. Typical PL spectra, under c.w. excitation at $5.0 \mathrm{eV}$, are reported in Fig. 1(b), at various temperatures ranging from $T=300 \mathrm{~K}$ to $T=4.5 \mathrm{~K}$. The PLE spectra of both PL emissions are centered at $\approx 5.1 \mathrm{eV}$ and their shapes are quite similar to each other and reproduce the $B_{2 \beta}$ absorption profile.

The strict relationship between the $\alpha_{E}$ and $\beta$ PL emissions and the OA band $B_{2 \beta}$ is evident in Fig. 2(a), where the sum of the two integrated PL intensities is plotted against the area of the OA band for the whole set of samples, at $T$ $=300 \mathrm{~K}$. Moreover, the ratio between the integrated intensities of the two PL emissions is sample independent [Fig. 2(b)]. The linear correlation between the intensities of the three bands, experimentally found in so large a variety of materials, confirms the hypothesis that the overall type- $B$ activity originates from the same point defect. In this scheme the $\alpha_{E}$ emission is interpreted as caused by the inverse transition $S_{1} \rightarrow S_{0}$, with a Stoke shift of about $0.9 \mathrm{eV}$, whereas the $\beta$ emission is ascribed to the $T_{1} \rightarrow S_{0}$ transition, excited through the ISC mechanism that populates the triplet state $T_{1}$. These band assignments are confirmed as well by the values of the decay constants, namely few ns for $\alpha_{E}$ (see below) and about $100 \mu$ s for $\beta$ band. ${ }^{8}$

Finally, we report that we did not find any correlation between the intensity of the overall type- $B$ activity and other known properties of the silica types investigated, such as $[\mathrm{OH}]$ content, bubble class, homogeneity, etc.

\section{B. Temperature dependence}

\section{The stationary PL emission}

The experimental data reported in Fig. 1(b) show that, on lowering $T$ from $T=300 \mathrm{~K}$ to $T=4.5 \mathrm{~K}$, the intensity of the $\alpha_{E}$ emission increases whereas that of the $\beta$ band decreases.

This thermal behavior can be related to the temperaturedependent effectiveness of the ISC process from $S_{1}$ to $T_{1}$. As a first approximation, we assume that $k_{\text {ISC }}$ varies with $T$ following the Arrhenius law: $k_{\mathrm{ISC}}(T)=k_{0} \exp \left[-H / K_{B} T\right]$, where $K_{B}$ is the Boltzmann constant, $H$ is the activation energy of the ISC process and $k_{0}$ is the pre-exponential factor. So, the ratio $\eta$ of the integrated intensities of the two bands is expected to depend on $T$ as

$$
\eta=\frac{k_{S}}{k_{0}} \exp \left(H / K_{B} T\right)
$$

Our experimental results are compared to Eq. (6) in Fig. 3, where we report experimental values of the parameter $\eta$ as a function of $1 / T$, as obtained in four different samples. We note that the simple Arrhenius law (a straight line in the used scales) accounts for the experimental results only in the high-
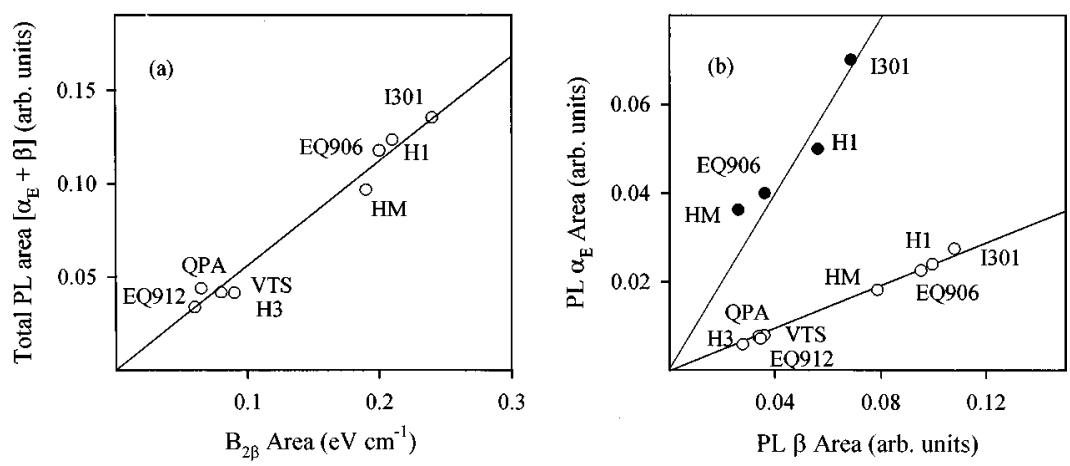

FIG. 2. (a) Correlation between the total PL emission bands at 3.1 and $4.2 \mathrm{eV}$ and the integrated intensity of the $\mathrm{OA}$ band $B_{2 \beta}$ at $5.15 \mathrm{eV}$; (b) Relationship between the two PL bands, at $300 \mathrm{~K}$ (open symbols) and at $220 \mathrm{~K}$ (full symbols). 


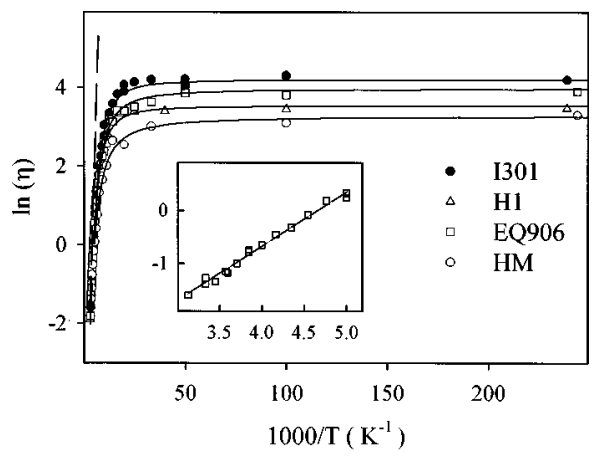

FIG. 3. Thermal behavior of the ratio between the integrated intensity of the $\alpha_{E}$ and $\beta$ bands. Symbols refer to: $\bullet, \mathrm{I} 301 ; \triangle, \mathrm{H} 1$; $\square$, EQ906 and $\bigcirc$, HM samples. The solid lines result from a fit of Eq. (7) to the experimental data in the whole temperature range 4.5-350 K. Broken line results from a fit of Eq. (6) (pure Arrhenius law with $H=0.08 \mathrm{eV}$ ) to the experimental data, in the temperature range 200 to $320 \mathrm{~K}$ (inset); in this case only the fit relative to the EQ906 sample data is reported, for the sake of clarity.

temperature range. This behavior is better evidenced in the inset reporting data for the sample EQ906 in a magnified scale. A best-fit procedure, in the range $200 \mathrm{~K} \leqslant T \leqslant 320 \mathrm{~K}$, yields $H=0.08 \pm 0.01 \mathrm{eV}$ and $k_{S} / k_{0}=0.01 \pm 0.005$ for all the samples. At variance, the Arrhenius law does not fit the data for $T \leqslant 200 \mathrm{~K}$ and $\eta$ tends to a constant value for $T$ $\leqslant 120 \mathrm{~K}$, so indicating that the ISC process becomes temperature independent.

For the quantitative analysis of the temperature dependence of the $k_{\text {ISC }}$, we follow the procedure described in Ref. 19, based on the modified Arrhenius law

$$
\eta=\frac{k_{S}}{k_{0}} \exp \left(H / K_{B} T_{\text {eff }}\right),
$$

where the effective temperature $T_{\text {eff }}$ is defined as

$$
T_{\text {eff }}=\frac{T_{f}}{1+\exp \left[\frac{T-T_{f}}{\Delta T}\right]}+\frac{T}{1+\exp \left[\frac{T_{f}-T}{\Delta T}\right]}
$$

and $T_{f}$ and $\Delta T$ are phenomenological parameters.

We recall that the above definition is heuristic and implies the presence of the so-called conformational substates, which have been successfully used in connection with other frustrated systems, e.g., proteins. ${ }^{20-21}$ In this picture, the active centers experience slightly different environments and each environment can rearrange in a variety of configurations, yielding different local energy minima (conformational substates). Transitions from a substate to another (interconversion) can occur via temperature-dependent processes. At high temperatures, interconversion is so frequent that the overall system is adequately described by a mean value $H$, $T_{\text {eff }}$ equals $T$ and $k_{\text {ISC }}$ obeys the conventional Arrhenius law. On the other limit, at low-temperature $\left(T \ll T_{f}\right)$ interconversion processes are frozen and each substate recovers its own specificity, namely its value $H_{i}$ of $H, T_{\text {eff }}$ tends to level to $T_{f}$ and $\eta$ levels to a constant value. So, $T_{f}$ and $\Delta T$ have the physical meaning of freezing point and of transition width, respectively.
TABLE I. Values of the parameters obtained by fitting Eq. (8) to the thermal evolution of the ratio between the emission integrated intensity of $\alpha_{E}$ and $\beta$ PL bands ( $\eta$ in the text).

\begin{tabular}{ccllc}
\hline \hline & $\mathrm{Ln}\left(k_{S} / k_{0}\right)$ & $T_{f}(K)$ & $\Delta T(K)$ & $H(\mathrm{eV})$ \\
\hline I301 & $-4.5 \pm 0.3$ & $126 \pm 5$ & $103 \pm 9$ & $0.07 \pm 0.02$ \\
H1 & $-4.4 \pm 0.3$ & $138 \pm 15$ & $115 \pm 8$ & $0.07 \pm 0.02$ \\
HM & $-4.7 \pm 0.1$ & $138 \pm 5$ & $100 \pm 30$ & $0.07 \pm 0.02$ \\
EQ906 & $-4.6 \pm 0.5$ & $133 \pm 10$ & $123 \pm 20$ & $0.07 \pm 0.02$ \\
\hline \hline
\end{tabular}

This interpretation scheme accounts for the experimental data reported in Fig. 3 also quantitatively. Full lines in Fig. 3 plot the Eqs. (7)-(8) that best fit the data and in Table I we list the best fit values of $T_{f}, \Delta T$, and $H$. As shown we get the same value of $H=0.07 \pm 0.02 \mathrm{eV}$ for all the samples, which nearly equals the value obtained by considering only the Arrhenius-like region. We obtain also similar values of $T_{f}(\sim 130 \mathrm{~K})$ and $\Delta T(\sim 110 \mathrm{~K})$ in all the samples.

As a conclusive comment, we remark that departures from the simple Arrhenius law could be alternatively explained in terms of a static distribution of the activation energies. However, in the case considered here, if one limits to consider bell-shaped distributions centered at the mean value $H_{\text {mean }}$ this model fails to account for both the leveling of $\eta$ at low $T$ and the fair Arrhenius behavior in the wide hightemperature range (shown in the inset of Fig. 3).

\section{Transient PL}

According to the model in Sec. II, when the exciting light is turned off, the PL emissions $\alpha_{E}$ and $\beta$ decay to zero following the single exponential laws, given by Eqs. (4)-(5). According to literature data, ${ }^{8} k_{T}$ is of the order of $10^{4} \mathrm{~s}^{-1}$, nearly independent on $T$. The decay of the $\alpha_{E}$ emission is reported in Fig. 4, as measured in our sample I301, at room and at low temperatures, under excitation at $5.0 \mathrm{eV}$.

At $T=10 \mathrm{~K}$, a single exponential law with characteristic time $\tau_{S}=7.3 \pm 0.1 \mathrm{~ns}$ fits well the experimental data. At this temperature, the ratio $k_{S} / k_{\mathrm{ISC}}$ is nearly 50 (from data in Fig. 3 and in Table I). So, the decay of the population $n_{S}$ from $S_{1}$ is essentially radiative and we can estimate $k_{S} \cong 1.4$ $\times 10^{8} \mathrm{~s}^{-1}$. On the other hand, the faster decay of curve (b) evidences the substantial contribution of the ISC process at room temperature. It is worth noting that at this temperature

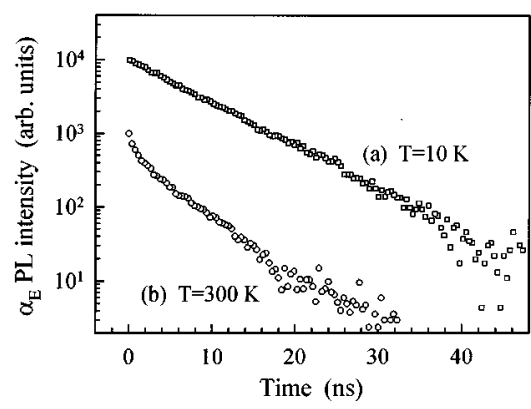

FIG. 4. Decay measurements of transient PL emission $\alpha_{E}$, with excitation at $5.0 \mathrm{eV}$, as detected in our sample $\mathrm{I} 301$ at $10 \mathrm{~K}$ (a) and at $300 \mathrm{~K}$ (b). For viewing purposes, the initial values of the decay curves are scaled by decades. 


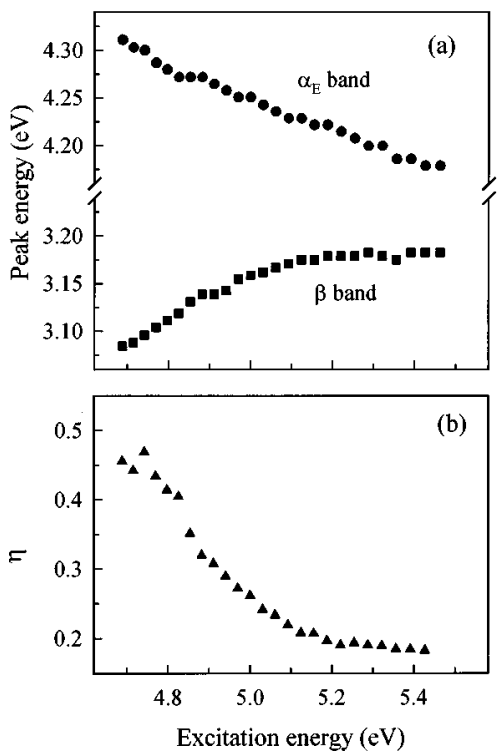

FIG. 5. Energy peak values of the PL spectra for the $\alpha_{E}$ and $\beta$ bands (a) and efficiency of the ISC mechanism, as measured by the $\eta$ ratio values (b), as a function of the excitation energy, at $T$ $=300 \mathrm{~K}$.

the decay is not a single exponential, which indicates a wide distribution of $k_{\text {ISC }}$ rates. More quantitatively, the initial decay time of the $\alpha_{E}$ emission ( $\tau_{i n}=0.9 \pm 0.1 \mathrm{~ns}$ ) allows to estimate the upper limit of the distribution of $k_{\text {ISC }}, \approx 1.1$ $\times 10^{9} \mathrm{~s}^{-1}$, higher than $k_{S}$ by a factor 8 . Moreover, from the slope of the final part of curve (b) we can derive that the lower limit for $k_{\text {ISC }}$ is less than $2 \times 10^{8} \mathrm{~s}^{-1}$. Therefore, at room temperature, the decay from $S_{1}$ appears to be strongly affected by ISC processes.

We note that the stationary and transient PL data give complementary information on the ISC process. In fact, the stationary PL data in the low-temperature range show the presence of conformational substates of each defect. At high temperature (from 300 to $200 \mathrm{~K}$ ), as evidenced by the Arrhenius behavior, a rapid interconversion among different substates occurs and, consequently, a single mean- $H$ value can be associated to the overall system. On the other hand, the PL transient measurements at $T=300 \mathrm{~K}$ evidences a distribution of $k_{\text {ISC' }}$, that at $T=10 \mathrm{~K}$ cannot be observed because of the poor efficiency of the ISC processes. On the basis of the previous point, this distribution is essentially the one related to the preexponential factors $k_{0}$ 's.

\section{Landscape of the excitation pathway}

The PL emission spectra reported in the previous section refer to the excitation condition $E_{\mathrm{Exc}}=5.0 \mathrm{eV}$. Here, we examine the effect of varying $E_{\text {Exc }}$ within the OA band $B_{2 \beta}$, namely in the range $4.6 \div 5.6 \mathrm{eV}$. In Fig. 5(a), we report the energy values $E_{\max }$ of the maxima of the two PL emissions as a function of $E_{\mathrm{Exc}}$ at $T=300 \mathrm{~K}$. As shown, both bands are shifted on varying $E_{\mathrm{Exc}}$. However, the effect is opposite for the two bands, as a blueshift of the $\beta$ band and a redshift of the $\alpha_{E}$ occur on increasing $E_{\text {Exc }}$.

In Fig. 5(b) we report the ratio $\eta$ as a function of excitation energy, at $T=300 \mathrm{~K}$. As can be seen, $\eta$ decreases (at



FIG. 6. The PLE spectra of the $\alpha_{E}$ (a) and $\beta$ (b) bands measured at three different emission energies around the maximum of each PL band: (a) Dotted line, $4.51 \mathrm{eV}$; continuous line, $4.26 \mathrm{eV}$; broken line, $4.07 \mathrm{eV}$. (b) Dotted line, $3.07 \mathrm{eV}$; continuous line, $3.16 \mathrm{eV}$; broken line, $3.32 \mathrm{eV}$.

least by a factor of 2) on increasing $E_{\mathrm{Exc}}$, indicating that the ISC efficiency is strongly dependent on the excitation energy.

Finally, a complementary effect is observed in the PLE spectra of the two bands, when they are revealed at different emission energies $E_{\mathrm{Em}}$. Typical results are reported in Figs. 6(a) and 6(b) where the PLE spectra, measured at three different values of $E_{\mathrm{Em}}$, are reported for the $\alpha_{E}$ and $\beta$ band, respectively. As shown, on increasing $E_{\mathrm{Em}}$, the PLE bandwidths increase and the peak positions shift, but, once again, in opposite directions for the two bands.

A qualitative interpretation of the dependence of the PL spectra on the excitation energy and of the PLE spectra on the emission one, can be put forward by hypothesizing a distribution of the centers, differing from each other not only for the energy distance from the ground and the excited states, but also for the ISC rate $k_{\mathrm{SC}}$. In considering the origin of observed bandwidths, we note that the values of the kinetic decay constants, which are of order of $\cong 10^{4} \mathrm{~s}^{-1}$ for $k_{T}$ and $\cong 10^{8} \mathrm{~s}^{-1}$ for $k_{S}$, allow us to exclude that the radiative decay contributes in a significant way to the observed broadening of about $0.25 \mathrm{eV}\left(\cong 6 \times 10^{13} \mathrm{~s}^{-1}\right)$. Therefore, so large bandwidths are to be related to the coupling with vibrational modes and to the conformational heterogeneity of the PL centers.

The coupling of the electronic transitions with the local modes of the matrix is relevant in determining the band shape and the energy peak positions. ${ }^{22-23}$ The large energy difference $(\Delta E \approx 0.9 \mathrm{eV}$ ) between the absorption band, at $\cong 5.15 \mathrm{eV}$, and the inverse transition ( $\alpha_{E}$ band), at $\cong 4.2 \mathrm{eV}$, reveals a large rearrangement of the surrounding nuclei after the point defect has undergone the transition from the excited to the ground state. Moreover, the temperature dependence of the spectral moments of the PL bands in different silica samples ${ }^{6}$ suggests that the vibrational coupling is a relevant effect in this type of materials. However, these coupling mechanisms do not explain the observed dependence of the 
spectral properties on $E_{\text {Exc }}$, reported in Figs. 5-6, since their effect on the intrinsic fine structure of emission bands should be essentially independent of the excitation energy.

The above reported results can be accounted only by a conformational heterogeneity in the surroundings of the point defects, that maps into the spectral broadening of the PL bands. In particular, the experimental data reported in Figs. 5-6 suggest the following remarks.

(i) The shift of the PL spectra in Fig. 5(a) indicates that, on increasing $E_{\text {Exc }}$, we explore active centers having lower and lower energy difference between the $S_{0}$ and $S_{1}$ states $\left(E_{s}\right)$ and higher and higher energy difference between the $S_{0}$ and $T_{1}$ states $\left(E_{T}\right)$. Moreover, according to the experimental data in Fig. 5(b), a significant increase of the efficiency of the ISC process occurs on increasing $E_{\mathrm{Exc}}$ indicating that the inhomogeneous distribution of the active centers involves not only the distance between the energy levels but also the values of $k_{\text {ISC }}$. Since the ISC mechanism is expected to depend on the local vibrational modes, these results indicate that the spectral distribution of the active centers is determined not only by the electronic properties of the chromophores but also by their interaction with the dynamics of the embedding matrix.

(ii) According to the PLE spectra reported in Fig. 6, at each emission energy we collect contributions coming from spectrally distinguishable centers, whose distribution is narrower and narrower and shifts in peak position on increasing $E_{\mathrm{Em}}$.

We note that in principle the reported data could be also interpreted in terms of a finite number of overlapping emission/excitation bands. ${ }^{13,24-25}$ However, in view of the fact that all the dependencies upon excitation energy are monotone, we consider more reliable that the excitation light explores a continuous distribution of centers that are slightly different both in the energy of the electronic states and in the interactions with the local dynamics.

\section{FINAL REMARKS}

The argument of the conformational disorder in glassy matrix is particularly relevant in the study of point defects in silica, both as grown and induced by irradiation, contributing to understand the wide spread of experimental results in samples of different origin and ascribed to structurally similar centers.

Based on the above reported data, we can speculate on the attribution of the observed spectral features to the presence in pure as grown natural silica samples of oxygen deficient defects localized on a Ge impurity. This last consideration, in fact, appears suitable by considering that the network of silica atoms can be largely distorted in the surrounding of the $\mathrm{Ge}$ atom, giving rise to conformational disorder.

Accordingly, data not reported here on silica samples containing centers responsible for the $A$-type activity, that are thought as oxygen point defects on a $\mathrm{Si}$ atom, show that $\alpha_{1}$ band profile is essentially independent upon the excitation energy. Moreover, the thermal behavior of the spectral moments of the PL emission bands of type- $A$ centers, both as grown and induced by $\gamma$ irradiation, in comparison with the ones of centers responsible of $B$-type activity, speaks for a larger interaction of the latter with vibrational modes of the vitreous matrix. Both considerations suggest that the point defects localized on a $\mathrm{Ge}$ atom experience a more distorted local environment with respect to the defect localized on $\mathrm{Si}$ atom. In our opinion, if this hypothesis will be confirmed also by further results, the spectral features related to the $\alpha_{E}$ and $\beta$ PL bands, together with those relative to the OA $B_{2 \beta}$ band, can be thought as a marker for the presence of $\mathrm{Ge}$ impurity of the order of $1: 10^{6}$ parts in as grown silica samples and can contribute to clarify the structural properties of defects induced, for example, by $\gamma$ irradiation.

\section{ACKNOWLEDGMENTS}

We wish to thank Mr. G. Lapis and Mr. F. D'Anca for technical assistance. This work was a part of a National research project supported by the Ministero Italiano della Ricerca Scientifica $e$ Tecnologica, Roma, Italy. The synchrotron radiation experiments were carried out at HASYLAB (DESY, Hamburg) (Project No. I-96-08 EC, Project Leader Professor G. Spinolo).
${ }^{1}$ L. N. Skuja, J. Non-Cryst. Solids 239, 16 (1998).

${ }^{2}$ R. Boscaino, M. Cannas, F. M. Gelardi, and M. Leone, Phys. Rev. B 54, 6194 (1996).

${ }^{3}$ R. Boscaino, M. Cannas, F. M. Gelardi, and M. Leone, Nucl. Instrum. Methods Phys. Res. B 116, 373 (1996).

${ }^{4}$ R. Boscaino, M. Cannas, F. M. Gelardi, and M. Leone, J. Phys. C 8, L545 (1996)

${ }^{5}$ M. Cannas, R. Boscaino, F. M. Gelardi, and M. Leone, J. NonCryst. Solids 216, 99 (1997).

${ }^{6}$ M. Leone, M. Cannas, and F. M. Gelardi, J. Non-Cryst. Solids 234, 514 (1998).

${ }^{7}$ R. Tohmon, H. Mizuno, Y. Ohki, K. Sasagane, K. Nagasawa, and Y. Hama, Phys. Rev. B 39, 1337 (1989).

${ }^{8}$ L. N. Skuja, J. Non-Cryst. Solids 149, 77 (1992).

${ }^{9}$ L. N. Skuja, J. Non-Cryst. Solids 167, 229 (1994).

${ }^{10}$ K. Awazu, H. Kawazoe, and K. Muta, J. Appl. Phys. 70, 69 (1991).
${ }^{11}$ H. Hosono, Y. Abe, D. L. Kinser, K. Muta, and H. Kawazoe, Phys. Rev. B 46, 11445 (1992).

${ }^{12}$ F. Meinardi and A. Paleari, Phys. Rev. B 58, 3511 (1998).

${ }^{13}$ A. Anedda, R. Boscaino, M. Cannas, R. Corpino, F. M. Gelardi, and M. Leone, Nucl. Instrum. Methods Phys. Res. B 116, 360 (1996).

${ }^{14}$ M. Leone, R. Boscaino, M. Cannas, and F. M. Gelardi, J. NonCryst. Solids 216, 105 (1997).

${ }^{15}$ J. R. Lakowicz, Principles of Fluorescence Spectroscopy (Plenum, New York, 1983).

${ }^{16}$ Quartz and Silice, Nemours, France, Catalogue OPT-91-3.

${ }^{17}$ Heraeus Quartzglas, Hanau, Germany, Catalogue POL-0/102/E.

${ }^{18}$ TSL Group PLC, Wallsend, England, Optical Products Catalogue.

${ }^{19}$ H. Gilch, R. Schweitzer-Stenner, W. Dreybrodt, M. Leone, A. Cupane, and L. Cordone, Int. J. Quantum Chem. 59, 301 (1996). 
${ }^{20}$ H. Frauenfelder, F. Parak, and R. D. Young, Annu. Rev. Biophys. Biophys. Chem. 17, 451 (1988).

${ }^{21}$ F. Parak and U. Nienhaus, J. Non-Cryst. Solids 131, 362 (1991).

${ }^{22}$ J. J. Markham, Rev. Mod. Phys. 31, 956 (1959).

${ }^{23}$ A. Cupane, M. Leone, E. Vitrano, and L. Cordone, Eur. Biophys.
J. 23, 349 (1994).

${ }^{24}$ M. Martini, F. Meinardi, A. Paleari, G. Spinolo, and A. Vedda, Phys. Rev. B 57, 3718 (1998).

${ }^{25}$ A. Anedda, C. M. Carbonaro, R. Corpino, and A. Serpi, J. NonCryst. Solids 216, 19 (1997). 